\title{
Proteomic profiling of large myofibrillar proteins from dried and long-term stored polyacrylamide gels
}

\author{
Sandra Murphy, Kay Ohlendieck* \\ Department of Biology, Maynooth University, National University of Ireland, Maynooth, Ireland
}

\section{A R T I C L E I N F O}

\section{Keywords:}

Gel drying

Gel electrophoresis

In-gel digestion

Mass spectrometry

Proteomics

Skeletal muscle

\begin{abstract}
A B S T R A C T
A method for the utilization of dried polyacrylamide gels from the pre-proteomic era is described in order to enable the mass spectrometric analysis of long-term stored protein preparations. The in-gel digestion of highmolecular-mass proteins embedded in a 20-year old gel was carried out following gel re-swelling and resulted in the proteomic identification of a large number of proteins, including $3400 \mathrm{kDa}$ titin, $800 \mathrm{kDa}$ nebulin and myosin heavy chains of $220 \mathrm{kDa}$ from rabbit skeletal muscle. These findings demonstrate that dried protein gels from past biochemical analyses can be successfully reused and analyzed by modern and refined mass spectrometric techniques.
\end{abstract}

One of the most frequently used biochemical methods for the efficient and rapid separation of complex protein samples is one-dimensional sodium dodecyl sulfate polyacrylamide gel electrophoresis, usually referred to as SDS-PAGE [1]. For the long-term preservation of polyacrylamide slab gels or for the usage of gels in autoradiography, gels are routinely dried and can then be conveniently stored at room temperature [2-4]. Prior to the development of large-scale and mass spectrometry-based proteomics [5], separated proteins were usually identified and characterized individually by peptide sequencing or labor-intensive immunochemical methods [6]. Since many bioanalytical research laboratories store large numbers of dried gels from the pre-proteomic era that have not undergone a more refined mass spectrometric analysis, it was of interest to investigate whether re-swelling of old gels followed by standardized in-gel digestion and routine mass spectrometric analysis would result in the identification of sensitive protein species, including very high-molecular-mass proteins.

Building on the findings from a select number of similar experiments that have previously been described for the proteomic analysis of medium size proteins from dried gels [7-11], this study has focused on the retrospective analysis of gel-embedded protein preparations by analyzing a subcellular fraction from a highly complex tissue. The bioanalytical approach was extended to extremely large protein species that are typically found in the contractile apparatus and its associated cytoskeletal network of skeletal muscle fibers. The main emphasis of this report was on sarcomeric proteins including titin, nebulin and myosin heavy chains, which exhibit molecular masses of approximately $3400 \mathrm{kDa}, 800 \mathrm{kDa}$ and $220 \mathrm{kDa}$, respectively [12,13]. In this brief note, we summarize the experimental workflow for the mass spectrometric identification of proteins recovered from dried and longterm stored polyacrylamide gels. Fig. 1 gives an overview of the individual steps involved in this proteomic study. For analytical details on mass spectrometry and bioinformatics, as well as listings of routinely used materials and chemicals, we would like to refer to recent publications from our laboratory on the standardized proteomic profiling of skeletal muscle proteins [14-16]. The approach described here falls under the general category of the GeLC-MS/MS method, which employs a combination of protein extraction, gel electrophoretic separation, ingel digestion, liquid chromatography and mass spectrometry for the unequivocal identification of individual protein species [17].

The subcellular fraction enriched in proteins from the myofibrils was isolated by a standardized procedure [18], and was originally separated 20 years ago on a $3-12 \%$ gradient SDS-PAGE gel [19]. Hind limb muscle tissue from adult New Zealand white rabbits was obtained as freshly dissected post-mortem specimens from the Bioresource Facility of the National University of Ireland. Rabbits were kept under standard conditions according to Irish legislation on the use of animals in experimental research. Transportation of samples to Maynooth University was carried out in accordance with the Department of Agriculture (animal by-product register number 2016/16 to the Department of Biology, National University of Ireland, Maynooth). For rabbit muscle protein separation, a Protean IIxi Cell system from BioRad Laboratories (Hemel Hempstead, Hertfordshire, UK) with 1.5$\mathrm{mm}$ thick and 16-cm long slab gels was used at a constant setting of $200 \mathrm{~V}$. Electrophoretic separation was carried out with $60 \mu \mathrm{g}$ protein per lane until the blue dye front had disappeared from the bottom of the gel, followed by protein staining with Coomassie Brilliant Blue.

\footnotetext{
* Corresponding author. Department of Biology, Maynooth University, National University of Ireland, Maynooth, Ireland.

E-mail address: kay.ohlendieck@mu.ie (K. Ohlendieck).
} 
Skeletal muscle tissue Tissue homogenization
Differential centrifugation

Myofibrillar fraction

$$
\begin{aligned}
& \text { Extraction } \\
& \text { Denaturation } \\
& \text { Reduction } \\
& \text { Alkylation }
\end{aligned}
$$

Distinct muscle protein population

Gel electrophoretic separation SDS-PAGE

\section{Gel with characteristic protein banding pattern}

Vacuum gel drying

\section{Dried protein gel for long-term storage}

\section{Characteristic peptide populations}

\section{Reconstituted muscle proteins}

In-gel digestion

Peptide extraction

Removal of contaminants

Desalting of peptides

Mass spectrometric analysis

LC-MS/MS

Bioinformatics analysis

\section{Identification of individual protein species}

Fig. 1. Flowchart of bioanalytical workflow used for the mass spectrometric identification of muscle proteins recovered from dried and long-term stored polyacrylamide gels.
Standard vacuum drying of the gel was carried out between two layers of acetate film in a solution of $30 \%$ methanol and 5\% glycerol in order to prevent cracking of the SDS-PAGE gel [4].

Following long-term storage in a lab book at room temperature, SDS-PAGE gels were re-swollen through overnight incubation at room temperature with gentle agitation in $30 \%$ methanol, $5 \%$ acetic acid and $5 \%$ glycerol. The following day gel strips were placed in fresh tubes and incubated at room temperature with shaking for $4 \mathrm{~h}$ in $10 \%$ glycerol and $1 \%$ acetic acid. The gel strips were subsequently incubated with $1 \%$ glycerol and $1 \%$ acetic acid overnight with shaking at room temperature. The gels were washed with 100\% MilliQ water and the acetate sheets were removed. Gel lanes with individual preparations were cut into 6 sections, and destained with $100 \mathrm{mM}$ ammonium bicarbonate/ acetonitrile $(1: 1, \mathrm{v} / \mathrm{v})$ shaking at room temperature for $30 \mathrm{~min}$, followed by incubation with $100 \%$ acetonitrile shaking at room temperature for $10 \mathrm{~min}$. In-gel digestion was performed with sequencing grade trypsin from Promega (Madison, WI, USA). The reconstituted protease was added to $50 \mathrm{mM}$ ammonium bicarbonate and each gel mixture treated at a 1:20 ration of trypsin to muscle protein overnight at $37{ }^{\circ} \mathrm{C}$ with agitation. Extraction buffer $(5 \%$ formic acid/acetonitrile $[1: 2, \mathrm{v} / \mathrm{v}]$ ) was added to gel pieces and incubated at $37{ }^{\circ} \mathrm{C}$ for $15 \mathrm{~min}$ and the supernatant fraction transferred to $1.5 \mathrm{ml}$ micro-centrifuge tubes and dried by vacuum centrifugation. Peptide populations were resuspended in $0.5 \%$ trifluoroacetic acid $/ 5 \%$ acetonitrile and centrifuged in $22 \mu \mathrm{m}$ acetate cellulose spin filter tubes for $20 \mathrm{~min}$ to remove any gel particles and then desalted using C18 spin columns (Thermo Fisher Scientific, Dublin, Ireland) and dried by vacuum centrifugation. Peptides were re-suspended in loading buffer consisting of $2 \%$ acetonitrile and $0.05 \%$ trifluoroacetic acid and analyzed by label-free liquid chromatography mass spectrometry (LC-MS/MS) using an Ultimate 3000 NanoLC system (Dionex Corporation, Sunnyvale, CA, USA) coupled to a Q-Exactive mass spectrometer (Thermo Fisher Scientific, Dublin, Ireland). The qualitative analysis of myofibrillar proteins was carried out with the Proteome Discoverer 1.4 against Sequest HT (SEQUEST HT algorithm, license Thermo Scientific, registered trademark University of Washington, USA) using the UniProtKB database with 23,072 proteins. Peptides were subsequently filtered using a minimum XCorr score of 1.5 for 1, 2.0 for $2,2.25$ for 3 and 2.5 for 4 charge states, with peptide probability set to high confidence.

The long-term stored SDS-PAGE gel with the myofibrillar fraction is

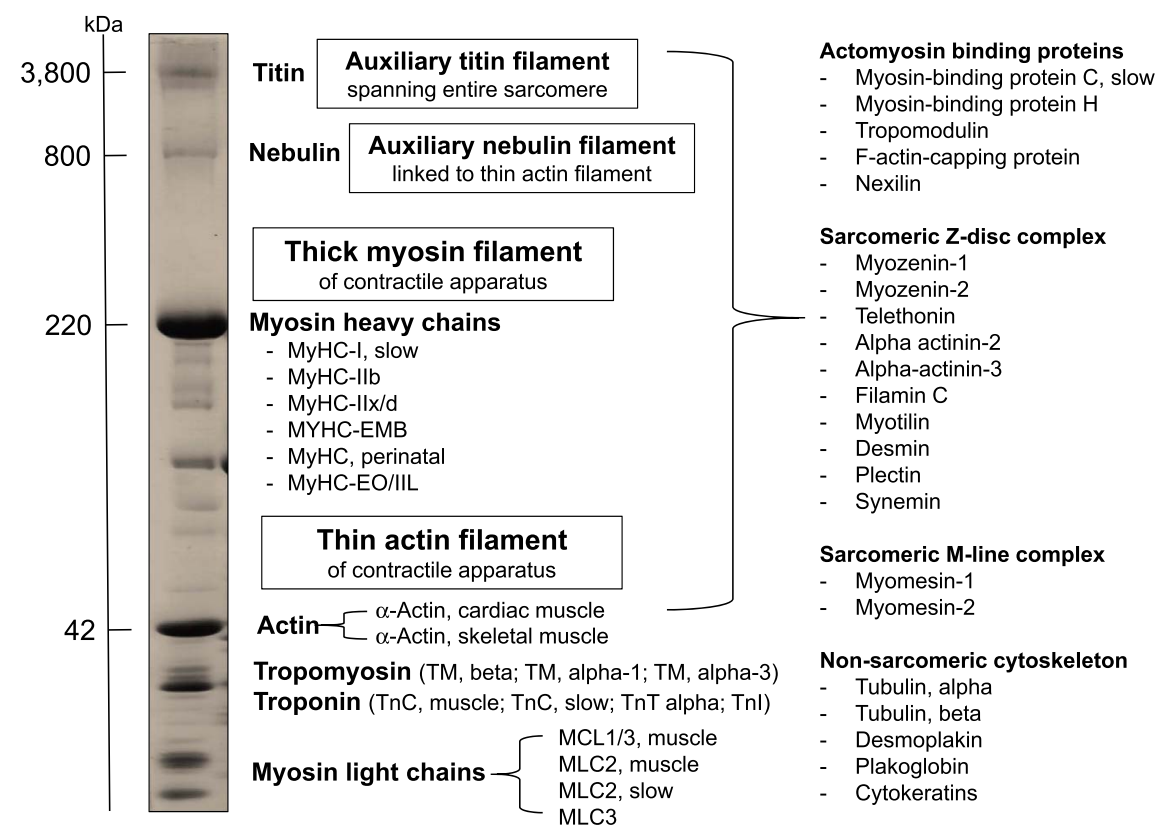

Fig. 2. Image of the long-term stored polyacrylamide gel that was used in this study to identify proteins in the myofibrillar fraction from rabbit skeletal muscle. The apparent molecular mass of the skeletal muscle marker proteins titin, nebulin, myosin heavy chain and actin is marked on the left of the gel. Included in the figure are key results from the proteomic identification of muscle proteins belonging to the contractile apparatus and its associated non-sarcomeric cytoskeleton. 
Table 1

List of major proteins of the contractile apparatus that were identified in the myofibrillar fraction from rabbit skeletal muscle by mass spectrometry-based proteomics.

\begin{tabular}{|c|c|c|c|c|c|}
\hline Protein name & Gene & $\%$ Coverage & Unique Peptides & Molecular mass (kDa) & $\mathrm{p} I$ \\
\hline Titin & TNT & 16.38 & 317 & 3451.0 & 6.23 \\
\hline Nebulin & NEB & 20.72 & 115 & 800.2 & 9.09 \\
\hline Myosin heavy chain MyHC-IIx/d (Myosin-1) & MYH1 & 53.50 & 28 & 223.4 & 5.80 \\
\hline Myosin heavy chain MYHC-EMB (Myosin-3) & MYH3 & 16.75 & 2 & 223.3 & 5.74 \\
\hline Myosin heavy chain MyHC-IIb (Myosin-4) & MYH4 & 47.06 & 20 & 223.1 & 5.80 \\
\hline Myosin heavy chain MyHC-IIb (Myosin-4) & MYH4 & 50.83 & 11 & 222.9 & 5.81 \\
\hline Myosin heavy chain MyHC-I/MyHC- $\beta$, slow/cardiac (Myosin-7) & MYH7 & 34.33 & 42 & 222.9 & 5.74 \\
\hline Myosin heavy chain MyHC, perinatal (Myosin-8) & MYH8 & 39.70 & 10 & 222.3 & 5.78 \\
\hline Myomesin-1 & MYOM1 & 8.67 & 13 & 186.5 & 5.99 \\
\hline Myomesin-2 & MYOM2 & 4.56 & 6 & 164.5 & 6.24 \\
\hline Myosin-binding protein C, slow-type & MYBPC1 & 8.03 & 8 & 119.1 & 6.05 \\
\hline Alpha-actinin-2 & ACTN2 & 21.48 & 13 & 103.8 & 5.52 \\
\hline Alpha-actinin-3 & ACTN3 & 22.09 & 13 & 102.4 & 5.45 \\
\hline Plectin & PLEC & 8.79 & 6 & 92.4 & 8.27 \\
\hline Myotilin & MYOT & 6.21 & 3 & 55.6 & 8.98 \\
\hline Myosin-binding protein $\mathrm{H}$ & MYBPH & 4.35 & 2 & 54.3 & 6.28 \\
\hline Desmin & DES & 34.54 & 16 & 53.4 & 5.27 \\
\hline Actin, alpha cardiac muscle & ACTC1 & 59.68 & 3 & 42.0 & 5.39 \\
\hline Actin, alpha skeletal muscle & ACTA1 & 59.68 & 3 & 42.0 & 5.39 \\
\hline Tropomodulin 4 & TMOD4 & 7.25 & 2 & 39.3 & 4.70 \\
\hline F-actin-capping protein subunit alpha-2 & CAPZA2 & 5.94 & 2 & 32.9 & 5.85 \\
\hline Tropomyosin beta chain & TPM2 & 65.49 & 12 & 32.8 & 4.70 \\
\hline Tropomyosin alpha-1 chain & TPM1 & 67.25 & 11 & 32.7 & 4.74 \\
\hline Myozenin-1 & MYOZ1 & 17.51 & 4 & 31.3 & 8.63 \\
\hline Tropomyosin & TPM & 41.30 & 2 & 28.9 & 4.81 \\
\hline Troponin $\mathrm{T}$ alpha isoform & TNNT2 & 22.22 & 7 & 27.8 & 9.54 \\
\hline Myosin light chain 3 & MYL & 25.36 & 4 & 23.1 & 5.19 \\
\hline Myosin light chain $1 / 3$, muscle isoform & MYL1 & 40.63 & 8 & 20.9 & 5.03 \\
\hline Troponin I (fragment) & TNNI2 & 26.26 & 5 & 20.9 & 9.20 \\
\hline Myosin light chain 2 & MYL2 & 40.00 & 2 & 19.0 & 4.92 \\
\hline Troponin C, slow skeletal and cardiac muscles & TNNC1 & 15.53 & 2 & 18.4 & 4.18 \\
\hline Troponin C, skeletal muscle & TNNC2 & 33.75 & 4 & 18.1 & 4.20 \\
\hline Myosin regulatory light chain 2 , muscle isoform 2 & MYLPF & 45.33 & 2 & 16.7 & 4.84 \\
\hline Alpha-tropomyosin, smooth muscle (fragment) & TPM1 & 51.61 & 2 & 14.1 & 4.67 \\
\hline
\end{tabular}

shown in Fig. 2, including key results from the proteomic identification of muscle marker proteins. Select findings from the mass spectrometric analysis of the isolated skeletal muscle fraction are listed in Table 1, including the high-molecular-mass proteins titin, nebulin and various isoforms of myosin heavy chain. In addition, major muscle protein bands of lower molecular mass are marked, including myosin light chains, troponins, tropomyosins and actins. Overall 164 proteins were identified in the myofibril fraction. The entire list of proteomic hits is summarized in Tables S1 in the Supplementary Information in the appendix of this note. Interestingly, a large number of distinct myosin heavy chains, i.e. slow MyHC-I, fast MyHC-IIb, fast MyHC-IIx/d, MYHCEMB, perinatal MyHC and MyHC-EO/IIL, and myosin light chains MCL1/3, slow MLC2, fast MLC2 and MLC3 were identified [13], demonstrating an excellent degree of sensitivity for protein identification in the long-term stored gel. In addition, both the muscle and cardiac isoform of $\alpha$-actin, as well as $\alpha$ - and $\beta$-tropomyosins and the troponin subunits TnI, TnC and TnT were identified in this study. As summarized in Fig. 2, key proteins belonging to the class of actomyosin binding proteins, sarcomeric Z-disk proteins, M-line complex proteins and cytoskeletal proteins [13] were also covered by the bioanalytical workflow outlined in this report. This shows that all major components of the contractile apparatus and its associated non-sarcomeric cytoskeleton were identified following the above described gel re-swelling, destaining and in-gel digestion procedure.

The decrease in overall yield of protein identification in freshly prepared versus long-term stored dried gels was estimated to be approximately $40 \%$, using the comparative proteomic screening of onedimensional gel slices containing microsomal membranes isolated from skeletal muscle homogenates (not shown). In relation to the detection of post-translation modifications in proteins following gel drying, as previously studied by Dihazi et al. [8], peptide files were searched for phosphorylation sites. Data analysis via Proteome Discoverer 1.4 using
Sequest HT followed by phosphoRS was carried out as recently described by Henry et al. [20]. Of the 2546 identified peptides, 27 contained phosphorylated sites and 24 of these peptides had a site probability score of $75 \%$ or greater for $\mathrm{S}$, $\mathrm{T}$, or $\mathrm{Y}$ amino acids. Phosphorylation sites in components of the contractile apparatus were identified in titin, plectin, cytokeratin and myosin heavy chain MyHCIIb.

In conclusion, the large number of identified muscle proteins from a 20-year old SDS-PAGE gel, including components of extremely high molecular mass such as the sarcomeric elements nebulin and titin, demonstrates that the described method can be utilized to re-use dried polyacrylamide gels following very long periods of storage at room temperature. Thus, stored gels from experiments carried out many years or even several decades ago during the pre-proteomic era can be successfully re-analyzed by more recently developed and highly sensitive mass spectrometry [21]. The straightforward combination of SDS-PAGE gel re-swelling, standardized in-gel digestion and routine LC-MS/MS analysis can now be employed to confirm and refine previous findings from gel-based studies, and might also be suitable for the retrospective proteomic profiling of scarce protein samples stored in dried gels. This might result in the identification of previously unknown protein factors and their involvement in biological or pathophysiological processes.

\section{Acknowledgements}

We thank Dr. Brendan Murray for the optimization of isolating the myofibrillar fraction from skeletal muscle. Research was supported by a Hume scholarship from Maynooth University and project grants from Muscular Dystrophy Ireland and the Irish Health Research Board (HRB/ MRCG-2016-20). The Q-Exactive quantitative mass spectrometer was funded under the Research Infrastructure Call 2012 by Science Foundation Ireland (SFI-12/RI/2346/3). 


\section{Appendix A. Supplementary data}

Supplementary data related to this article can be found at http://dx. doi.org/10.1016/j.ab.2017.11.022.

\section{References}

[1] B.J. Smith, SDS polyacrylamide gel electrophoresis of proteins, Methods Mol. Biol. 32 (1994) 23-34.

[2] M.L. Cheng, J.L. VandeBerg, A versatile technique for drying polyacrylamide slab gels, J. Biochem. Biophys. Methods 11 (1985) 137-143.

[3] B.B. Samal, Drying and storage of polyacrylamide slab gels: a simple procedure, Anal. Biochem. 163 (1987) 42-44.

[4] S. Stamova, I. Michalk, H. Bartsch, M. Bachmann, Gel drying methods, Methods Mol. Biol. 869 (2012) 433-436.

[5] R. Aebersold, M. Mann, Mass spectrometry-based proteomics, Nature 422 (2003) 198-207.

[6] H. Towbin, T. Staehelin, J. Gordon, Immunoblotting in the clinical laboratory, J. Clin. Chem. Clin. Biochem. 27 (1989) 495-501.

[7] H. Matsumoto, N. Komori, Protein identification on two-dimensional gels archived nearly two decades ago by in-gel digestion and matrix-assisted laser desorption ionization time-of-flight mass spectrometry, Anal. Biochem. 270 (1999) 176-179.

[8] H. Dihazi, R. Kessler, K. Eschrich, In-gel digestion of proteins from long-term dried polyacrylamide gels: matrix-assisted laser desorption-ionization time of flight mass spectrometry identification of proteins and detection of their covalent modification, Anal. Biochem. 299 (2001) 260-263.

[9] O. Panfilov, B. Lanne, Peptide mass fingerprinting from wet and dry two-dimensional gels and its application in proteomics, Anal. Biochem. 307 (2002) 393-395.

[10] J. Zhou, J. Li, J. Li, P. Chen, et al., Dried polyacrylamide gel absorption: a method for efficient elimination of the interferences from SDS-solubilized protein samples in mass spectrometry-based proteome analysis, Electrophoresis 31 (2010) 3816-3822.

[11] P.G. Righetti, B. Lomonte, J.J. Calvete, Resurrexit, sicut dixit, alleluia. Snake venomics from a 26-year old polyacrylamide focusing gel, J. Proteomics 75 (2012) 1074-1078.

[12] L.C. Meyer, N.T. Wright, Structure of giant muscle proteins, Front. Physiol. 4 (2013) 368.

[13] A. Holland, K. Ohlendieck, Proteomic profiling of the contractile apparatus from skeletal muscle, Expert Rev. Proteomics 10 (2013) 239-257.

[14] S. Carberry, M. Zweyer, D. Swandulla, K. Ohlendieck, Comparative proteomic analysis of the contractile-protein-depleted fraction from normal versus dystrophic skeletal muscle, Anal. Biochem. 446 (2014) 108-115.

[15] S. Murphy, M. Zweyer, R.R. Mundegar, et al., Concurrent label-free mass spectrometric analysis of dystrophin isoform Dp427 and the myofibrosis marker collagen in crude extracts from mdx-4cv skeletal muscles, Proteomes 3 (2015) 298-327.

[16] S. Murphy, P. Dowling, M. Zweyer, et al., Proteomic analysis of dystrophin deficiency and associated changes in the aged $\mathrm{mdx}-4 \mathrm{cv}$ heart model of dystrophinopathy-related cardiomyopathy, J. Proteomics 145 (2016) 24-36.

[17] J.A. Paulo, Sample preparation for proteomic analysis using a GeLC-MS/MS strategy, J. Biol. Methods 3 (2016) pii: e45.

[18] J. Gannon, P. Doran, A. Kirwan, K. Ohlendieck, Drastic increase of myosin light chain MLC-2 in senescent skeletal muscle indicates fast-to-slow fibre transition in sarcopenia of old age, Eur. J. Cell Biol. 88 (2009) 685-700.

[19] B.E. Murray, K. Ohlendieck, Cross-linking analysis of the ryanodine receptor and alpha1-dihydropyridine receptor in rabbit skeletal muscle triads, Biochem. J. 324 (1997) 689-696.

[20] M. Henry, M. Power, P. Kaushik, et al., Differential phosphoproteomic analysis of recombinant Chinese hamster ovary cells following temperature shift, J. Proteome Res. 16 (2017) 2339-2358.

[21] A.S. Deshmukh, M. Murgia, N. Nagaraj, et al., Deep proteomics of mouse skeletal muscle enables quantitation of protein isoforms, metabolic pathways, and transcription factors, Mol. Cell. Proteomics 14 (2015) 841-853. 